\title{
Magnetism of Nanowires Driven by Novel Even-Odd Effects
}

\author{
Samir Lounis, ${ }^{*}$ Peter H. Dederichs, and Stefan Blügel \\ Institut für Festkörperforschung, Forschungszentrum Jülich, D-52425 Jülich, Germany
}

(Received 27 November 2007; published 5 September 2008)

\begin{abstract}
The parity of the number of atoms in finite antiferromagnetic nanowires deposited on ferromagnets is shown to be a crucial quantity determining their magnetic ground state. Relating results of the fullpotential Korringa-Kohn-Rostoker method for noncollinear magnetism from first principles to a Heisenberg model, we show that the magnetic structure changes dramatically across the entire nanowire if one single atom is added to it. Infinite and finite even-numbered nanochains exhibit always noncollinear magnetism, while odd-numbered wires lead under given conditions to a collinear ferrimagnetic ground state. This extremely nonlocal effect occurs only for nanosized wires.
\end{abstract}

DOI: 10.1103/PhysRevLett.101.107204

Controlling the flow of charge and spin information to and from increasingly smaller becoming structures hinges on the meticulous control of the coupling between spins. Obviously, this is of central importance for the design of novel devices engineered at the level of individual atomic spins [1] whose functionality is geared towards computing speed, storage capacity, and energy saving. Fundamental explorations of magnetic nanowires and nanostructures using advanced experimental methods [2-6] opened up unprecedented opportunities for atomic engineering of future spintronics and quantum information devices.

Although a tremendous effort (see, e.g., Refs. [7-12]) has been undertaken to understand ferromagnetic clusters in general and ferromagnetic chains, in particular, little if nothing is known about the behavior of antiferromagnetic nano-objects. Because of inherent experimental and theoretical difficulties, the task of unraveling the complex magnetic behavior of antiferromagnetic nanostructures remains challenging. These experience frequently a phenomenon called magnetic frustration, which describes the inability to satisfy competing exchange interactions between neighboring atoms. It is partially removed by creating noncollinear structures with lower energies [13-17]. These structures can be of unprecedented complexity with magnetic moments aligned along quantization axes changing from atom to atom, and thus much more difficult to measure. Very recently, a breakthrough in this field has been achieved in investigating the magnetism of small chains of 1 to $10 \mathrm{Mn}$ atoms on a thin insulating layer using inelastic scanning tunneling microscopy [18].

The most evident examples of frustration is the so-called geometric frustration arising from the antiferromagnetic interaction between adatoms forming, e.g., a trimer with a shape of an equilateral triangle. In this Letter we present a novel frustration phenomenon due to the coupling with the ferromagnetic substrate: Below a particular size, nanowires exhibit totally different magnetic structures depending on the parity of the number of adatoms. Evennumbered chains of antiferromagnetic atoms reveal a noncollinear (NC) structure; however, odd-atom chains
PACS numbers: 75.75.+a, 73.22.-f, 73.63.Nm, 75.30.Hx

show a completely different magnetic order, a collinear ferrimagnetic (FI) structure. The latter fact is very surprising since both the dimer as well as the infinite chain have basically the same NC structure. In other words, the magnetic structure of the whole wire dramatically changes or switches if a single atom is added. We discovered that this extremely nonlocal phenomenon is a nanoeffect occurring only for nanowires below a certain length. The differences between the magnetic structures of the even and odd-atom wires are predicted to be sufficiently large to be observed experimentally. Throughout the Letter, results are shown primarily for $\mathrm{Mn}$ chains on $\mathrm{Ni}(001)$, but they are general and hold also for antiferromagnetic chains on different substrates.

The ground-state spin structure of nanowires with up to 11 adatoms is investigated with the full-potential screened Korringa-Kohn-Rostoker (KKR) Green function method $[13,19]$ based on density-functional theory (DFT) employing the local spin density approximation (LSDA) [20]. Angular momenta up to $l_{\max }=3$ were included in the expansion of the Green functions and up to $2 l_{\max }=6$ in the charge density expansion. First, the Green functions of the surface serving as the reference system are determined. Second, a cluster of perturbed potentials, which always includes the wire atoms and all nearest neighbors (NN) substrate atoms, is considered with a size varying from 20 up to 83 perturbed sites for all kind of ad-chains considered. Experience has shown that $\mathrm{Mn}$ adatoms on $\mathrm{Ni}(001)$ relax only by a small amount (less than $3 \%$ ) inwards. Thus, we consider the wire atoms at the unrelaxed hollow position. Within the cluster of embedded potentials, both the values and the directions of the magnetic moments are calculated [13].

Throughout this Letter, we shall use a Heisenberg model to interpret the results of the aforementioned densityfunctional calculations, to extend the results to larger chains and so provide an overall picture. We assume a simple classical spin-Hamiltonian in which magnetic exchange interactions between first-neighbor atoms are taken into account 


$$
H=-J_{1} \sum_{i=1}^{N-1} \cos \left(\theta_{i}-\theta_{i+1}\right)-J_{2} \sum_{i=1}^{N} \cos \left(\theta_{i}\right) .
$$

$N$ is the number of atoms in the chain and $\theta$ is the rotation angle of the wire atom moment with respect to the magnetization of the surface. The DFT calculations give tiny rotation angles for the Ni substrate moments and thus are neglected in the model. For symmetry reasons, the magnetic moments lie on a circle, not a sphere; i.e., $\theta$ ranges from 0 to $360^{\circ}$ with the azimuth $\phi=0^{\circ}$. The magnetic exchange interactions $\left(J_{1}\right.$ and $\left.J_{2}\right)$ are extracted from our $a b$ initio calculations [21] and inserted into the present model: $J_{1}(<0)$ stands for an (antiferromagnetic) exchange interaction between two neighboring wire atoms at sites $i$ and $i \pm 1$ in the chain, while $J_{2}$ is the total magnetic exchange interaction between a given wire atom and its neighboring surface atoms. Typically, the parameters $J_{1}$ and $J_{2}$ describe the ab initio results well and dominate over interactions between further neighbors or higher order interactions. For Ni substrates there is a small variation in the value of $J_{2}$ due to the breathing of the length of the $\mathrm{Ni}$ moments upon $\theta$, which is neglected in the model.

$A b$ initio results of the dimer and trimer:-The simplest case of an antiferromagnetic (AF) chain, a dimer (Cr or $\mathrm{Mn}$ ) deposited, for example, on a ferromagnetic surface such as $\mathrm{Ni}(001)$ was already studied in Ref. [13]. We recall here briefly the main results for $\mathrm{Mn}$ : Mn-dimer atoms couple strongly AF to each other $\left(J_{1}<0\right)$, which is in competition with the ferromagnetic (FM) interaction of Mn with the substrate atoms $\left(J_{2}>0\right)$ favoring a parallel alignment of the dimer moments. Thus, frustration in the interactions occurs and a NC structure [see Fig. 1(a)] is obtained as ground state. Here the Mn moments are aligned antiparallel to each other and roughly perpendicular to the substrate moments. Moreover, the weak FM interaction with the substrate causes a slight tilting of the moments leading to an angle $\theta$ of $73^{\circ}$ instead of $90^{\circ}$. Out of the six $\mathrm{NN} \mathrm{Ni(001)} \mathrm{substrate} \mathrm{moments,} \mathrm{the} \mathrm{four} \mathrm{outer} \mathrm{ones} \mathrm{show} \mathrm{a}$ small tilting of $7.4^{\circ}$, while the two inner ones do not tilt due to symmetry reasons.

For our preliminary discussion, we use the Heisenberg model [Eq. (1)]. For the dimer case, a potential FI solution depends only on $J_{1}\left(E_{\mathrm{FI}}=J_{1}\right)$, because the contributions $J_{2}$ of both adatoms cancel out due to their antiparallel alignment. On the other hand, the NC solution depends also on the magnetic interaction with the substrate in terms of $J_{2}\left[E_{\mathrm{NC}}=-J_{1} \cos (2 \theta)-2 J_{2} \cos (\theta)\right]$ being clearly the cause of the noncollinearity of the system as energy is gained by the upward tilting [Fig. 1(a)] of the moments from $90^{\circ}$ to $73^{\circ}$. For three Mn adatoms [Fig. 1(b)], we find the FI solution to be the ground state. Contrary to the dimer, the energy of the collinear solution of the trimer depends on $J_{2}\left(E_{\mathrm{FI}}=2 J_{1}-J_{2}\right)$ due to the additional third adatom, which in fact allows the FI solution to be the ground state.

One sees here the premise of an odd-even effect on the nature of the magnetic ground state. On this basis one might conjecture that wires with an even number of atoms would behave similarly to the dimer because an additional interaction energy with the substrate proportional to $J_{2}$ can only be gained in the $\mathrm{NC}$ state by the small tiltings off the $90^{\circ}$ angle shown in Fig. 1(a), while odd-numbered wires would behave similarly to the trimer. They can always gain energy in the collinear state due to one $J_{2}$ interaction term which does not cancel out.

$A b$ initio results of longer nanowires:-Investigating the longer nanochains with even number of atoms shows that their ground state is always NC. Examples are presented in Figs. 1(c), 1(e), 1(g), and 1(i) and in Table I. In first approximation, the magnetic moments are always in the plane perpendicular to the substrate magnetization keeping the magnetic picture seen for the dimer almost unchanged. Moreover, the neighboring magnetic moments are coupled almost AF. The atoms at both ends of the chains have the most rotated moments towards a FM coupling to the substrate moments (see Table I). The two central chain atoms A-B (see Fig. 1 for the notation) are the ones which keep
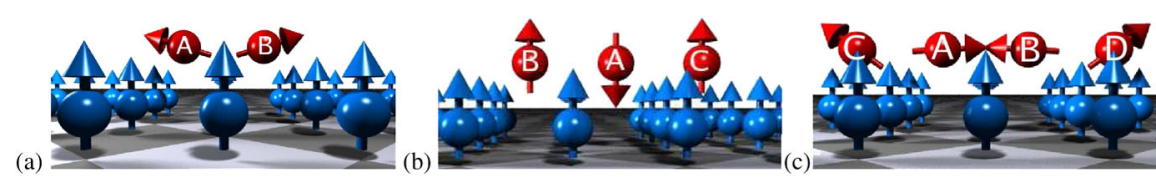
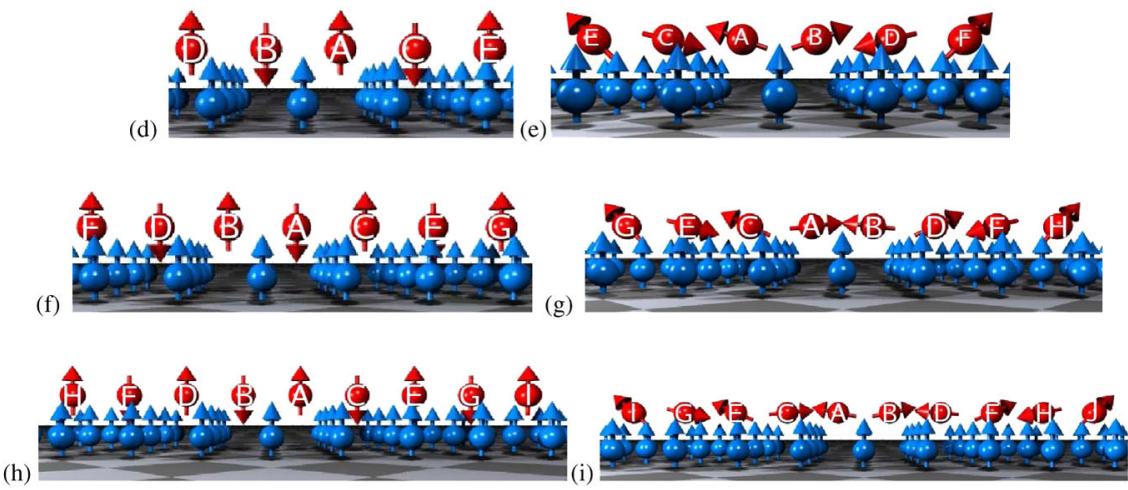

FIG. 1 (color online). Different magnetic ground configurations of the Mn nanowires on $\mathrm{Ni}(001)$. The nanochains with an even number of Mn atoms (2, 4, $6,8,10)$ prefer a noncollinear ground state, the odd ones a collinear one. 
TABLE I. $A b$ initio results for even-numbered nanochains: size and angle of the magnetic moments as well as total energy differences between the NC and FI solutions. In every pair of wire atoms connected by the character "-", the azimuthal angles $\phi$ are equal to $0^{\circ}-180^{\circ}$, while the magnetic moments and rotation angles $\theta$ are the same.

\begin{tabular}{ccccc}
\hline \hline $\begin{array}{c}\text { Length } \\
\text { (adatoms) }\end{array}$ & Adatom & $\theta\left(^{\circ}\right)$ & $M\left(\mu_{B}\right)$ & $\begin{array}{c}E_{\mathrm{NC}}-E_{\mathrm{FI}} \\
(\mathrm{meV} / \text { adatom })\end{array}$ \\
\hline 2 & A-B & 73 & 3.71 & -11.16 \\
4 & A-B,C-D & 87,54 & $3.55,3.72$ & -8.48 \\
6 & A-B, C-D, & 70,104, & $3.46,3.52$, & -7.82 \\
& E-F & 45 & 3.67 & \\
8 & A-B, C-D, & 84,65, & $3.47,3.46$, & -5.46 \\
& E-F, G-H & 106,44, & $3.52,3.66$ & \\
10 & A-B, C-D, & 78,85, & $3.47,3.47$ & -3.53 \\
& E-F, G-H, & 67,102, & $3.46,3.52$ & \\
& I-J & 48 & 3.67 & \\
\hline \hline
\end{tabular}

their rotation angles almost unaltered with respect to the dimer. The modulus of the angle $\theta$ oscillates between $70^{\circ}$ obtained for the chain with 6 atoms up to $87^{\circ}$ obtained for the chain with 4 atoms. Note that the angle between two successive moments is around $150^{\circ}$ similar to the dimer result.

The considered odd-numbered nanochains are characterized by a FI ground state in which the majority of atoms are coupled FM to the surface. The total energy differences to the lowest lying metastable, i.e., NC state, first increases with respect to the length of the wire (see Table II) up to a maximum for a wire with 7 atoms $(10.48 \mathrm{meV} /$ adatom) followed by a decrease for longer wires. This behavior is the property of the metastable NC state and arises from a competition between the edge and inner atoms of the chain. Edge atoms in odd chains favor collinear moment alignment to the substrate. For short chains, trimer and 5 atoms chains, they dominate the total magnetic behavior permitting only a slight tilting of the moments away from the FI state. For longer wires, however, the inner atoms experience basically the same local environment as the atoms in even chains accompanied by similar moment orientations.

TABLE II. $A b$ initio results for odd-numbered nanochains: size of the magnetic moments and total energy differences between the NC and FI state. As described earlier the atoms separated by the character "-" have the same magnetic moment.

\begin{tabular}{cccc}
\hline \hline $\begin{array}{c}\text { Length } \\
\text { (adatoms) }\end{array}$ & Adatom & $M\left(\mu_{B}\right)$ for FI & $\begin{array}{c}E_{\mathrm{NC}}-E_{\mathrm{FI}} \\
(\mathrm{meV} / \text { adatom })\end{array}$ \\
\hline 3 & A, B-C & $-3.78,3.65$ & 8.85 \\
5 & A, B-C, D-E & $3.43,-3.56,3.64$ & 9.52 \\
7 & A, B-C, D-E, & $-3.54,3.43,-3.56$ & 10.48 \\
5 & F-G & 3.64 \\
& A, B-C, D-E, & $3.43,-3.54,3.43$ & 9.29 \\
11 & F-G, H-I & $-3.56,3.64$ \\
\hline \hline
\end{tabular}

When increasing the length of the chains, both kinds of wires should converge to the same magnetic ground state since the parity of the chains is expected to be an obsolete quantity for infinite chains. Within the DFT framework, the investigation of longer chains is computationally very demanding. Thus, in the next section we will use the afore described Heisenberg model to investigate this magnetic transition.

Heisenberg model results:-Two different approaches are used to solve Eq. (1). At first, we allow the rotation angle $\theta_{i}$ to vary from site to site in the chain and at second, we consider a constant absolute value of $\theta$ at each site. The first, the inhomogeneous approach, requires an iterative numerical scheme while the second, the homogeneous one, leads to a simple analytical form. In Fig. 2, the energy difference between the NC and FI states determined by the first approach is plotted versus the length of Mn nanowires. Negative values refer to a $\mathrm{NC}$ ground state. The model confirms the DFT results showing that the even chains have always a NC ground state. Within this model, the ground state for odd wires changes from FI to $\mathrm{NC}$ when the number of atoms exceeds a transition length of 9 atoms which is smaller than what predicted from DFT. Moreover, even beyond this length, we notice an oscillatory behavior of the energy differences and the magnetic structure when going from the odd- to even-numbered chains. This behavior is easier to understand when considering the homogeneous ansatz, which leads to a somewhat bigger transition length (19 atoms) for the odd chains. In this case, the energy difference per wire atom is given by $\Delta E_{\mathrm{NC}-\mathrm{FI}}=$ $\frac{N J_{2}^{2}}{8(N-1) J_{1}}+P(N) \frac{\left|J_{2}\right|}{N}$, with $P(N)$, a parity function, equals 0 when $N$ is even, or 1 if $N$ is odd. Since the first term of $\Delta E_{\mathrm{NC}-\mathrm{FI}}$ is negative for all lengths $\left(J_{1}<0\right), \mathrm{NC}$ is the ground state for all even chains. The second term, on the other hand, is positive and provides for finite lengths an energy counterbalance allowing the FI the lowest energy

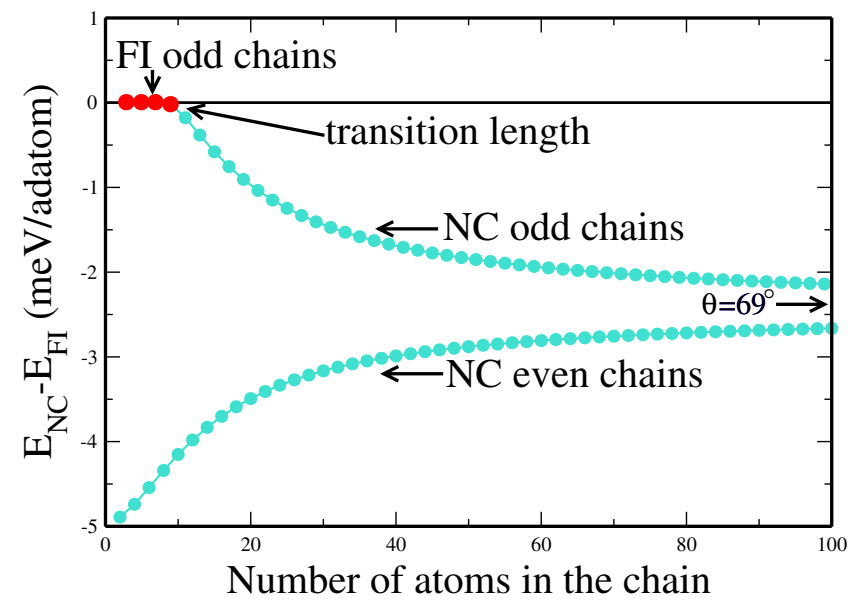

FIG. 2 (color online). Energy differences obtained from the Heisenberg model between the $\mathrm{NC}$ and the FI configuration for different lengths of $\mathrm{Mn}$ wires on $\mathrm{Ni}(001) . J_{1}$ and $J_{2}$ are, respectively, equal to -138.2 and $52 \mathrm{meV}$. 


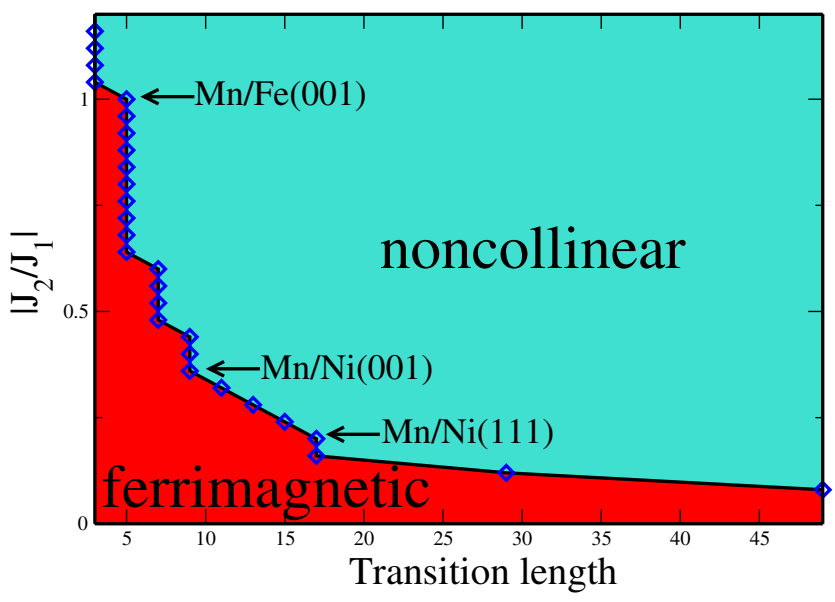

FIG. 3 (color online). Magnetic phase diagram of the odd chains showing the effect of the ratio $J_{2} / J_{1}$ on the transition lengths. If the length of the chains is smaller than the transition length, the FI configuration is the ground state.

solution. As this term decreases as $N^{-1}$, a crossover to noncollinearity is expected for $N \approx 8\left|J_{1}\right| /\left|J_{2}\right|$ as the preferred behavior for large lengths, as found in Fig. 2. Moreover, we notice that for big values of $N, \Delta E_{\mathrm{NC}-\mathrm{FI}}$ converges to a constant, $J_{2}^{2} /\left(8 J_{1}\right)$, which is confirmed by the convergence of the two curves in Fig. 2 towards the same NC state with $\theta_{\mathrm{NC}}=69^{\circ}:$ if the chains are infinite the parity induced differences vanish.

The next point is the discussion of the general behavior of the transition length for odd chains. Using the inhomogeneous ansatz, we determine for each set of parameters $\left(J_{1}, J_{2}\right)$ the corresponding transition length which leads to the phase diagram shown in Fig. 3. The obtained curve seems to decay roughly as $N^{-1}$. On one hand, small exchange interactions ratios lead to very long transition lengths. This means that odd-even effects are expected to last for very long chains which can be easily observed experimentally. On the other hand, big values of $J_{2}$ compared to $J_{1}$ lead to very small transition lengths. The obtained phase diagram is interesting and can be used to predict the behavior as well as the transition lengths for other kinds of AF chains deposited on FM substrates. This model predicts, for example, a transition length of 5 atoms for $\mathrm{Mn} / \mathrm{Fe}(001)$ while $\mathrm{Mn} / \mathrm{Ni}(111)$ is characterized by a value of 17 atoms. Certainly, this transition length is subject to modifications depending on the accuracy of the exchange interactions, spin-orbit coupling, and geometrical relaxations. Furthermore, we point out that the angles $\theta$ obtained by the model are in good agreement with our DFT calculations, meaning that the model reliably describes the very-long chains. In addition, it is interesting to note that a recent experimental as well as theoretical work revealed a similar NC behavior for a full monolayer of Mn deposited on a bcc Fe(001) surface [22].

To conclude, using DFT calculations, a strong novel phenomenon was found for finite antiferromagnetic nanowires on ferromagnetic substrates by which the parity of the number of atoms in a wire is an essential quantity determining the magnetic structure. Even-numbered nanochains exhibit a NC ground state, nanosized odd-numbered chains below a particular length have a collinear ground state. Adding one atom to the chain can lead to a complete change of the magnetic structure across the entire chain elucidating the extreme nonlocality of the effect. A Heisenberg model fitted to our ab initio results allows us to interpret these results and to determine transition for various chain-substrate combinations characterizing the odd chains. Finally, we predict the infinite chains to be of noncollinear magnetic nature. We encourage experimental efforts to investigate this intriguing parity effect.

We thank Dr. Mavropoulos for fruitful discussions. This work was supported by the ESF EUROCORES Programme SONS under Contract No. ERAS-CT-2003-980409 and the DFG Priority Programme SPP1153.

*s.lounis@fz-juelich.de

[1] I. Žutić, J. Fabian, and S. Das Sarma, Rev. Mod. Phys. 76, 323 (2004).

[2] Y. Yayon et al., Phys. Rev. Lett. 99, 067202 (2007).

[3] T. Mirkovic et al., Nature Nanotech. 2, 565 (2007).

[4] S. Rusponi et al., Nature Mater. 2, 546 (2003); W. Kuch, ibid. 2, 505 (2003).

[5] J. A. Stroscio and R. J. Celotta, Science 306, 242 (2004).

[6] J. T. Lau et al., Phys. Rev. Lett. 89, 057201 (2002).

[7] A. Smogunov et al., Nature Nanotech. 3, 22 (2008).

[8] P. Gambardella et al., Science 300, 1130 (2003).

[9] B. Lazarovits, L. Szunyogh, P. Weinberger, and B. Újfalussy, Phys. Rev. B 68, 024433 (2003).

[10] Y. Mokrousov, G. Bihlmayer, S. Heinze, and S. Blügel, Phys. Rev. Lett. 96, 147201 (2006).

[11] J. Lagoute, C. Nacci, and S. Folsch, Phys. Rev. Lett. 98, 146804 (2007).

[12] O. Sipr et al., J. Phys. Condens. Matter 19, 096203 (2007).

[13] S. Lounis, Ph. Mavropoulos, P.H. Dederichs, and S. Blügel, Phys. Rev. B 72, 224437 (2005); S. Lounis et al., ibid. 75, 174436 (2007); S. Lounis et al., Europhys. Lett. 81, 47004 (2008).

[14] A. Bergman et al., Phys. Rev. B 73, 174434 (2006).

[15] R. Robles and L. Nordström, Phys. Rev. B 74, 094403 (2006).

[16] S. Uzdin, V. Uzdin, and C. Demangeat, Europhys. Lett. 47, 556 (1999).

[17] A. T. Costa, R. B. Muniz, and D. L. Mills, Phys. Rev. Lett. 94, 137203 (2005).

[18] C. F. Hirjibehendin, C.P. Lutz, and J. Heinrich, Science 312, 1021 (2006); A. J. Heinrich et al., Science 306, 466 (2004).

[19] K. Wildberger, R. Zeller, and P. H. Dederichs, Phys. Rev. B 55, 10074 (1997), and references therein.

[20] S. H. Vosko, L. Wilk, and M. Nusair, Can. J. Phys. 58, 1200 (1980).

[21] A. I. Liechtenstein, M. I. Katsnelson, V.P. Antropov, and V. A. Gubanov, J. Magn. Magn. Mater. 67, 65 (1987).

[22] C. Grazioli, D. Alfè, S. R. Krishnakumar, et al., Phys. Rev. Lett. 95, 117201 (2005). 